\title{
Diálogo sobre una Ecuación Cúbica ${ }^{1}$
}

\author{
Luis Báez Duarte ${ }^{\dagger}$, Alfredo Octavio
}

In Memoriam: Dedicado a la memoria de Luis Báez Duarte, con quien escribiera estas lineas hace un tiempo. Alfredo Octavio.

\section{Resumen}

En este artículo se presenta, en forma de diálogo, una forma novedosa de obtener las soluciones de una ecuación cúbica.

Palabras y frases clave: ecuación cúbica, ecuación cuadrática, resolvente, diálogo,

\section{Dialogue about a cubic equation}

\begin{abstract}
In this paper it is presented a novel way to obtain the solutions of a cubic equation.
\end{abstract}

Key words and phrases: cubic equation, quadratic equation, resolvent, dialogue .

\section{Diálogo}

(Dos matemáticos frente a una pizarra trabajan en un problema...)

AO: Bueno, hasta aqui llegó esta sesión, hay que encontrar una raíz de esta ecuación cúbica. Tendremos que ir a la biblioteca.

LBD: ¿Por qué?

AO: Para buscar la fórmula, i¿o me vas a decir que tú te la sabes de memoria?!

LBD: De memoria no, pero la podemos deducir.

AO: ¡Perderemos mucho tiempo!

LBD: ¿Sabes deducir la resolvente de una ecuación cuadrática? Es decir, si te doy el polinomio.

$$
a x^{2}+b x+c=0
$$

Recibido 25/05/2019. Aceptado 25/07/2019. 
cómo demuestras que

$$
x=\frac{-b \pm \sqrt{b^{2}-4 a c}}{2 a} ?
$$

AO: Eso es fácil. Si dividimos entre a y llamamos a las raíces $\alpha$ y $\beta$, tendríamos que

$$
x^{2}+\frac{b}{a} x+\frac{c}{a}=(x-\alpha)(x-\beta)=x^{2}-(\alpha+\beta) x+\alpha \beta .
$$

Entonces obtenemos que la suma de las raíces debe ser $-b / a$ y el producto $c / a$. Es decir,

$$
\begin{aligned}
\alpha+\beta & =-\frac{b}{a} \\
\alpha \beta & =\frac{c}{a}
\end{aligned}
$$

Y ahora, usando un poco de sentido común...

LBD: ¡El menos común de los sentidos!

AO: Partiendo de (1), fíjate que la distancia de los números $\alpha$ y $\beta$ a su punto medio $-\frac{b}{2 a}$ es la misma, hay un número $d$ que cumple $\alpha=-\frac{b}{2 a}+d$ y $\beta=-\frac{b}{2 a}-d$. Sustituyendo esto en la segunda ecuación, obtenemos

$$
\left(-\frac{b}{2 a}+d\right)\left(-\frac{b}{2 a}-d\right)=-\frac{b^{2}}{4 a^{2}}-d^{2}=\frac{c}{a},
$$

de donde

$$
d^{2}=\frac{b^{2}}{4 a^{2}}-\frac{c}{a}=\frac{b^{2}-4 a c}{4 a^{2}}
$$

Como ya encontramos $d$, podemos calcular $\alpha$ y $\beta$ y obtenemos la conocida fórmula de la resolvente de una ecuación cuadrática:

$$
x=\frac{-b \pm \sqrt{b^{2}-4 a c}}{2 a}
$$

LBD: Bueno, ya te lo dije, el truquito de buscar un $d$ de esa manera no parece tan de sentido común, sino bien ingenioso, pero muy bien, ahora, ¿por qué no haces lo mismo para la ecuación cúbica?

AO: ¡Imposible! 
LBD: Es fácil si recuerdas otro truco que me enseñó, cuando ya era un niño, mi tío, el matemático venezolano Francisco J. Duarte. Es totalmente inolvidable. Empecemos por recordar el cubo de una suma, escribámoslo de una manera especial:

$$
(\alpha+\beta)^{3}=\alpha^{3}+3 \alpha^{2} \beta+3 \alpha \beta^{2}+\beta^{3}=3 \alpha \beta(\alpha+\beta)+\left(\alpha^{3}+\beta^{3}\right)
$$

Esto quiere decir que la ecuación cúbica de forma

$$
x^{3}-3 \alpha \beta x-\left(\alpha^{3}+\beta^{3}\right)=0
$$

siempre tiene una raíz $x=\alpha+\beta$. Ahora una ecuación cúbica general

$$
a x^{3}+b x^{2}+c x+d=0
$$

Siempre se puede llevar a la forma

$$
y^{3}+p x+q=0
$$

¿Sabes cómo hacerlo?

AO: Creo que sí. Dividir la ecuación (3) entre a no es problema, luego sustituyendo $x$ por $y-\frac{b}{3 a} y$ queda de la forma pedida de la ecuación (4).

LBD: ¡Correcto! ¿Y estás de acuerdo en que si encuentro una raíz de esta última ecuación (4), puedo encontrar una raíz de la ecuación original (3)?

AO: Claro, el proceso es completamente reversible.

LBD: Pero por lo que decámos antes sólo hay que encontrar $\alpha$ y $\beta$ tales que

$$
\begin{aligned}
\alpha^{3}+\beta^{3} & =q, \\
\alpha \beta & =\frac{p}{3} .
\end{aligned}
$$

De aquí podemos obtener el siguiente sistema, que te debe resultar conocido...

$$
\begin{aligned}
\alpha^{3}+\beta^{3} & =q, \\
\alpha^{3} \beta^{3} & =\frac{p^{3}}{27} .
\end{aligned}
$$

AO: ¡Si conoces la suma y el producto de dos números puedes saber cuáles son los números!

LBD: Exacto, usando lo que tú mismo explicabas hace un rato para la ecuación de segundo grado, podemos calcular $\alpha^{3}$ y $\beta^{3}$, de donde podemos encontrar la raíz $\alpha+\beta$. Si lo necesitáramos podríamos calcular las otras dos raíces. 
AO: Sí, podríamos dividir los polinomios...

LBD: Bueno, hagamos los cálculos para la ecuación que obtuvimos y continuemos trabajando un rato más.

AO: OK. Pero pronto vamos a almorzar.

LBD: ¡Oye vale, siempre pensando en la comida!

Luis Báez Duarte

Alfredo Octavio (aoctavio@tekeno.com)

Tekeno 